\title{
Beyond spectral tuning: human cone visual pigments adopt different transient conformations for chromophore regeneration
}

Sundaramoorthy Srinivasan 1

Arnau Cordomí 2

Eva Ramon 1

Pere Garriga $1, *$

Phone +34937398568

Email pere.garriga@upc.edu

1 Departament d'Enginyeria Química, Centre de Biotecnologia

Molecular, Universitat Politècnica de Catalunya, Rambla de Sant Nebridi 22, 08222 Terrassa, Spain

2 Laboratori de Medicina Computacional, Unitat de Bioestadística, Facultat de Medicina, Universitat Autònoma de Barcelona, Cerdanyola del Vallès, 08193 Barcelona, Spain

\section{Abstract}

Human red and green visual pigments are seven transmembrane receptors of cone photoreceptor cells of the retina that mediate color vision. These pigments share a very high degree of homology and have been assumed to feature analogous structural and functional properties. We report on a different regeneration mechanism among red and green cone opsins with retinal analogs using UV-Vis/fluorescence spectroscopic analyses, molecular modeling and site-directed mutagenesis. We find that photoactivated green cone opsin adopts a transient conformation which regenerates via an unprotonated Schiff base linkage with its natural chromophore, whereas red cone opsin forms a typical protonated Schiff base. The chromophore regeneration kinetics is consistent with a secondary retinal uptake by the cone pigments. Overall, our findings reveal, for the 
first time, structural differences in the photoactivated conformation between red and green cone pigments that may be linked to their molecular evolution, and support the proposal of secondary retinal binding to visual pigments, in addition to binding to the canonical primary site, which may serve as a regulatory mechanism of dark adaptation in the phototransduction process.

AQ1

\section{Keywords}

Color vision

Ligand binding

Visual phototransduction

Retinal

G-protein coupled receptors

Abbreviations

9CR 9-cis-Retinal

11CR 11-cis-Retinal

ATR All-trans-retinal

DM $n$-Dodecyl-b $\boldsymbol{\beta}$-D-maltoside

GPCR G-protein coupled receptor

ECL Extracellular loop

PBS Phosphate buffered saline

PSB Protonated Schiff base

SB Schiff base

TM Transmembrane

Electronic supplementary material

The online version of this article (doi:10.1007/s00018-015-2043-7) contains supplementary material, which is available to authorized users.

\section{Introduction}

Human color perception is mediated by cone photoreceptor cells which are mainly located in the fovea of the retina $[1,2]$. Bright light activates the photosensitive opsin pigments which are embedded in the outer segment membrane discs of cone retinal cells thereby initiating the complex process of 
photopic vision with a fast response [3]. The cone visual pigments correspond to blue opsin (SWL, short wavelength), green opsin (MWL, medium wavelength) and red opsin (LWL, long wavelength). In the genome, the red cone opsin gene $(O P N 1 L W)$ is arranged proximal to a green cone opsin gene $(O P N 1 M W)$ in an array at Xq28 with $98 \%$ identical nucleotide sequences [4, 5 ] presenting a $96 \%$ protein sequence identity and differing merely in 15 amino acids. This very high sequence identity has prompted the assumption that both green and red pigments share the same structural and functional features. The blue pigment, however, shows a lower degree of $43 \%$ homology to the red and green opsins [5]. Thus, aside from their specific role in spectral tuning into different absorption wavelengths, the differences in structurefunction relationships caused by this minor sequence variance have not been previously elucidated. Furthermore, most of the mechanistic insights into cone phototransduction are extrapolated from the rod photoreceptor cell system, responsible for dim-light scotopic vision, and its visual pigment rhodopsin which has been much more extensively studied [6].

The cone opsin pigments belong to the superfamily of G-protein-coupled receptors (GPCRs) consisting of a heptahelical transmembrane apoprotein and a photosensitive chromophore, the vitamin A derivative 11-cis-retinal (11CR). In its inactive dark-adapted state, the retinal chromophore is covalently bound to these visual pigments through its aldehyde group via a protonated Schiff base (PSB) linkage to the $\varepsilon$-amino group of Lys ${ }^{7.43}$ (numbering corresponding to $\mathrm{red} /$ green human opsins, with the Ballesteros-Weinstein general scheme [7] as superscript) in the seventh transmembrane (TM7) helix. The PSB is stabilized by a salt bridge with the carboxylic group of the counterion, Glu129 $9^{3.28}$ at TM3 [8]. A PSB is a structural feature of visual pigments and its corresponding visible spectrum would show an absorption maximum at $440 \mathrm{~nm}$. However, the characteristic differences in visual sensitivities are due to specific amino acid variations in the retinal binding pocket which result in different absorption maxima - as a result of the so-called opsin shift effect - corresponding to $560 \mathrm{~nm}$ for the red cone pigment $530 \mathrm{~nm}$ for the green cone pigment, and $420 \mathrm{~nm}$ for the blue cone pigment [9-11].

The first step in the phototransduction process involves photon absorption by the visual pigments that causes $11 \mathrm{CR}$ isomerization to all-trans-retinal (ATR) [12] leading to the formation of active metarhodopsinII (MetaII) conformation which binds and activates the G-protein transducin [13]. Photoactivation of visual pigments alters the $\mathrm{pKa}$ of the PSB and induces deprotonation which is 
the primary molecular event of the visual transduction cascade after retinal isomerization [14, 15]. Subsequent breakage of the PSB linkage results in retinal release from the retinal binding pocket. The MetaII conformation of cone opsins is short lived compared to that of rhodopsin [16-19].

In a recent report on the detailed comparison of photoactivated properties of rhodopsin and red cone opsin, we could conclude that there is a difference in retinal analog accessibility in the regeneration process of red cone opsin and rhodopsin [20] which was proposed to be relevant to the fine-tuning of ligand-receptor interactions in GPCRs [21]. We have now extended our approach by comparing the structural and functional features of the closely related red and green cone opsins upon photoactivation. To this aim we have focused on the detailed analysis of the regeneration ability, and subsequent dark adaptation, of red and green cone opsins with retinal analogs immediately after illumination, including the kinetics of retinal binding and release in the presence of exogenously added retinal.

\section{Materials and methods}

\section{Materials}

The red and green cone opsin genes, cloned into pMT4 plasmid vector, were kindly provided by Prof. Kevin D. Ridge. Dulbecco's modified Eagle medium (PAA Laboratories), supplemented with fetal bovine serum (Sigma), L-glutamine (Sigma), and penicillin-streptomycin (Sigma), was used to culture COS-1 cells (American Type Culture Collection no. CRL-1650). 11CR was provided by the National Eye Institute, National Institutes of Health. Purified mAb rho-1D4 was obtained from Cell Essentials and was coupled to CNBr-activated Sepharose beads (Sigma). $n$-Dodecyl- $\beta$-D-maltoside (DM) was purchased from Affymetrix. The nonamer-peptide H-TETSQVAPA-OH was obtained from Unitat de Tècniques Separatives i Síntesi de Pèptids, Universitat de Barcelona. 9CR, hydroxylamine, protease inhibitor cocktail, and phenylmethanesulfonyl fluoride (PMSF) were purchased from Sigma, and polyethyleneimine (PEI) was purchased from Polysciences. Quartz absorption cuvettes with light path $10 \mathrm{~mm}$ (Hellma Analytics, Germany).

Oligonucleotides were purchased from Sigma (Spain).

\section{Expression and purification of red and green cone pigments}

Red, or green, cone opsin was expressed in transiently transfected COS-1 
cells by chemical transfection using PEI reagent. Cells were harvested 48-60 $\mathrm{h}$ after transfection and regenerated with $10 \mu \mathrm{M}$ 11CR in PBS buffer (137 mM NaCl, $2.7 \mathrm{mM} \mathrm{KCl}, 10 \mathrm{mM} \mathrm{Na}_{2} \mathrm{HPO}_{4}$, and $1.8 \mathrm{mM} \mathrm{KH}_{2} \mathrm{PO}_{4}, \mathrm{pH}$ 7.4) by overnight incubation at $4{ }^{\circ} \mathrm{C}$. Regenerated cells were subsequently solubilized using $1 \% \mathrm{DM}$ with PMSF and protease inhibitors, and the pigments were purified by immunoaffinity chromatography using Sepharose coupled to rho-1D4 antibody. The bound cone opsin pigments were eluted in PBS containing the nonamer peptide and $0.05 \% \mathrm{DM}$.

\section{UV-Vis spectrophotometry of cone pigments}

Purified cone pigments were spectroscopically characterized using a Varian Cary 100 Bio spectrophotometer (Varian), equipped with a water-jacketed cuvette holder connected to a circulating water bath. Temperature was controlled by a peltier accessory connected to the spectrophotometer and the temperature was maintained at $20{ }^{\circ} \mathrm{C}$ during all experiments. All spectra were recorded in the 250-650 $\mathrm{nm}$ range for green and 250-700 $\mathrm{nm}$ for red cone opsin, with a bandwidth of $2 \mathrm{~nm}$, a response time of $0.5 \mathrm{~s}$, and a scan speed of $400 \mathrm{~nm} / \mathrm{min}$.

\section{Fluorescence spectroscopy of cone pigments}

QuantaMaster 4 spectrofluorimeter (Proton Technology International) was employed to measure the Trp fluorescence emission corresponding to retinal release and uptake processes. The excitation wavelength was $295 \mathrm{~nm}$, and the emission wavelength was $330 \mathrm{~nm}$, measuring 1 point per second for $2 \mathrm{~s}$, followed by a 28 s pause (with a beam shutter to prevent sample photobleaching by the fluorimeter lamp). The excitation slit settings were $0.5 \mathrm{~nm}$, and emission was $10 \mathrm{~nm}$. Trp fluorescence was monitored over time in the dark until a steady baseline was obtained. Then the sample was photobleached ( $>495 \mathrm{~nm}$ ) for $30 \mathrm{~s}$ using a Dolan-Jenner MI-150 fiber-optic illuminator, and the change in fluorescence was recorded. For regeneration experiments, a 2.0- to 2.5-fold molar concentration of retinal over pigment was used which accounts for a maximum of $1 \%$ of the protein sample volume.

\section{Site-directed mutagenesis}

Phe309Tyr was introduced into human green cone opsin gene using site-directed mutagenesis kit (Quickchange, Stratagene) using the mutagenic primers: forward primer -5'GCCCTGCCGGCCTACTTTGCCAAAAGTGCC3', reverse primer -5'GGCACTTTTGGCAAAGTAGGCCGGCAGGGC-3'. 


\section{Molecular models of red and green opsin}

Homology models for the dark state and active human red and green opsin were constructed, respectively, based on the crystal structure of bovine rhodopsin (PDB id: 1GZM) [22] and the opsin soaked with ATR (PDB id: 2X72) [23]. Modeller 9.8 was used for this purpose [24]. All models were energy minimized using AMBER program [25], employing the Amberff99SB-ILDN forcefield [26].

We computed interaction potential maps [27] using them as implementation in MOE (Molecular Operating Environment (MOE), 2013.08; Chemical Computing Group Inc., 1010 Sherbooke St. West, Suite \#910, Montreal, QC, Canada, H3A 2R7, 2015.) in order to identify possible secondary binding pockets for retinal. Flexible docking of the second retinal molecule was done using AutoDock Vina [28].

\section{Results}

\section{Regeneration mechanism of green and red cone opsins with retinal analogs}

The dark-state UV-Vis spectrum of purified green cone pigment showed two characteristic bands with absorption maxima at 530 and $280 \mathrm{~nm}$ (Fig. 1 a, solid line) corresponding to the amount of regenerated green cone pigment present and the total opsin pigment, respectively. The regeneration experiment was carried out by adding an aliquot of a concentrated stock sample of $11 \mathrm{CR}$ yielding increased absorbance at $380 \mathrm{~nm}$ corresponding to the free $11 \mathrm{CR}$ added (Fig. 1 a, dotted line). No absorbance increase at $530 \mathrm{~nm}$ (Fig. $1 \mathrm{a}$, dashed line) was observed after illumination which suggested that the photoactivated conformation of green cone opsin was not able to regenerate with $11 \mathrm{CR}$. In contrast, a parallel experiment performed with 9CR, showed a regenerated band at $\sim 495 \mathrm{~nm}$ with $85 \%$ maximal regeneration (Fig. $1 \mathrm{~b}$ ).

\section{Fig. 1}

Regeneration of photoactivated green and red cone pigments with $11 \mathrm{CR}$ and 9CR. a, b Immunopurified human recombinant green cone pigment was analyzed by UV-visible spectrophotometry in the dark (1, solid line) and after addition of exogenous ligand, i.e., 2.5-fold of either 11CR (a) or 9CR (b) over opsin (2, dotted line). The pigment was then illuminated for $30 \mathrm{~s}(>495 \mathrm{~nm})$ and a spectrum was recorded after $30 \mathrm{~min}$ (3, dashed line). c, $\mathbf{d}$ Red cone opsin was spectroscopically measured in an analogous way to green cone opsin and spectra were measured in the dark (1, solid line), after 11CR (c) or 9CR (d) addition (2, 
dotted line), and 30 min after illumination (3, dashed line)
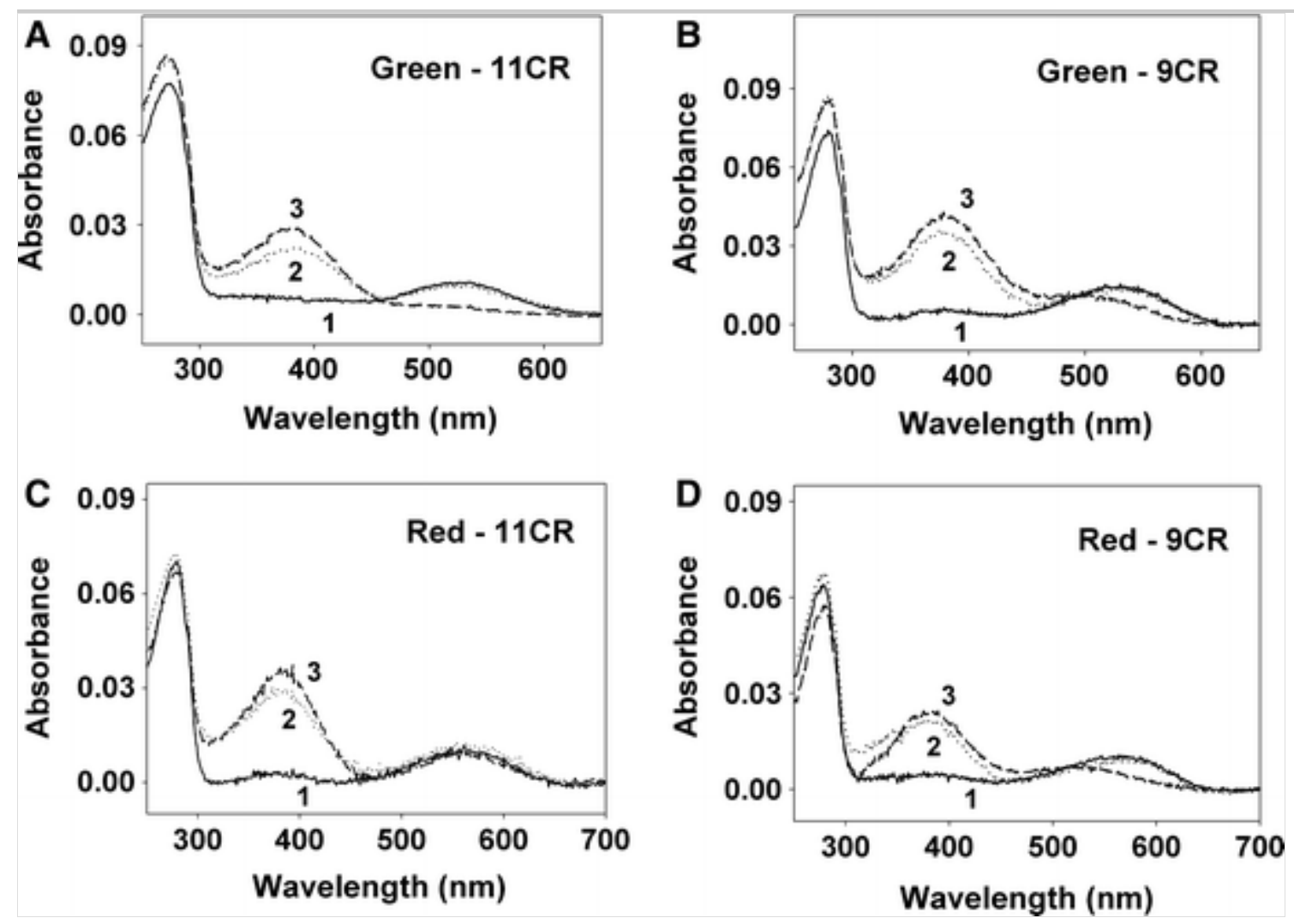

Photoactivated red cone opsin regenerates via a PSB with both 9CR and 11CR

An analogous regeneration experiment was carried out with purified red cone opsin. In both experiments, 9CR and 11CR can bind opsin after illumination and regenerate the visible chromophore with PSB linkage indicated by the presence of absorbance increase in the visible region above $440 \mathrm{~nm}$. In particular, complete regeneration with $11 \mathrm{CR}$ was achieved as indicated by the re-appearance of the typical absorption maximum at $560 \mathrm{~nm}$ (Fig. 1 c), whereas $\sim 70 \%$ regeneration with 9CR was recovered with an absorption maximum at $\sim 525 \mathrm{~nm}$ (Fig. 1d). The shifted absorption maximum of the protein is due to the specific absorbance properties of 9CR.

Photoactivated green cone opsin regenerates with 11CR by means of an unprotonated SB linkage

The lack of absorbance increase in the visible region suggested no $11 \mathrm{CR}$ binding and prompted the question whether green cone opsin could be regenerating with $11 \mathrm{CR}$ by means of an unprotonated SB linkage in which the absorption maximum of the regenerated pigment would be shifted to the UV region [29]. To test this hypothesis, two parallel UV-Vis spectroscopic experiments were carried out with the same green opsin sample that was 
illuminated and split into two aliquots. One of the aliquots was acidified immediately after photobleaching, whereas the other was acidified $30 \mathrm{~min}$ later (Fig. 2a). The difference spectrum between the two aliquots showed a band at $\sim 40 \mathrm{~nm}$ (Fig. $2 \mathrm{~b}$ ) which clearly indicated that the photoactivated green cone opsin regenerates via an unprotonated SB linkage with 11CR. The same experiment was carried out for red cone opsin, in which $11 \mathrm{CR}$ regeneration after illumination can be detected in the visible region, and sample acidification shifted the absorption band to $440 \mathrm{~nm}$ as well (Fig. S1). In order to rule out that the differences in regeneration mechanisms may be due to the different stability of red and green cone opsins in the detergent solution, the reactivity of dark-state red and green cone opsin pigments with hydroxylamine (which can access the cone opsin retinal binding site and sequester the bound chromophore to form retinaloxime) was tested (Fig. S2). Both pigments showed similar reaction kinetics indicating that differences in stability were not the cause of the observed different regeneration mechanisms.

\section{Fig. 2}

Photoactivated green cone opsin regenerates with $11 \mathrm{CR}$ by means of an unprotonated Schiff base linkage. a The UV-vis spectrum of purified green cone pigment was measured in the dark (1, solid line). After addition of 2.5 -fold $11 \mathrm{CR}$, it was illuminated for $30 \mathrm{~s}(\lambda>495 \mathrm{~nm})(2$, dotted line). Then, the sample was split into two aliquots in which one was acidified with $2 \mathrm{~N} \mathrm{H}_{2} \mathrm{SO}_{4}$ immediately (3, dashed line) and the other $30 \mathrm{~min}$ (4, dot-dashed line) later. b A difference spectrum was obtained by subtracting the spectrum of the sample acidified after $30 \mathrm{~min}$ and the sample acidified immediately after photoactivation. c Green cone pigment, in a fluorimetric micro-cuvette was illuminated for $30 \mathrm{~s}(\lambda>495 \mathrm{~nm})$ after a stable baseline was obtained. Then, 2.5 -fold of $11 \mathrm{CR}$ to the concentration of the pigment was added immediately after photoactivation and mixed thoroughly. The dotted line represents the fast regeneration process immediately after $11 \mathrm{CR}$ addition 

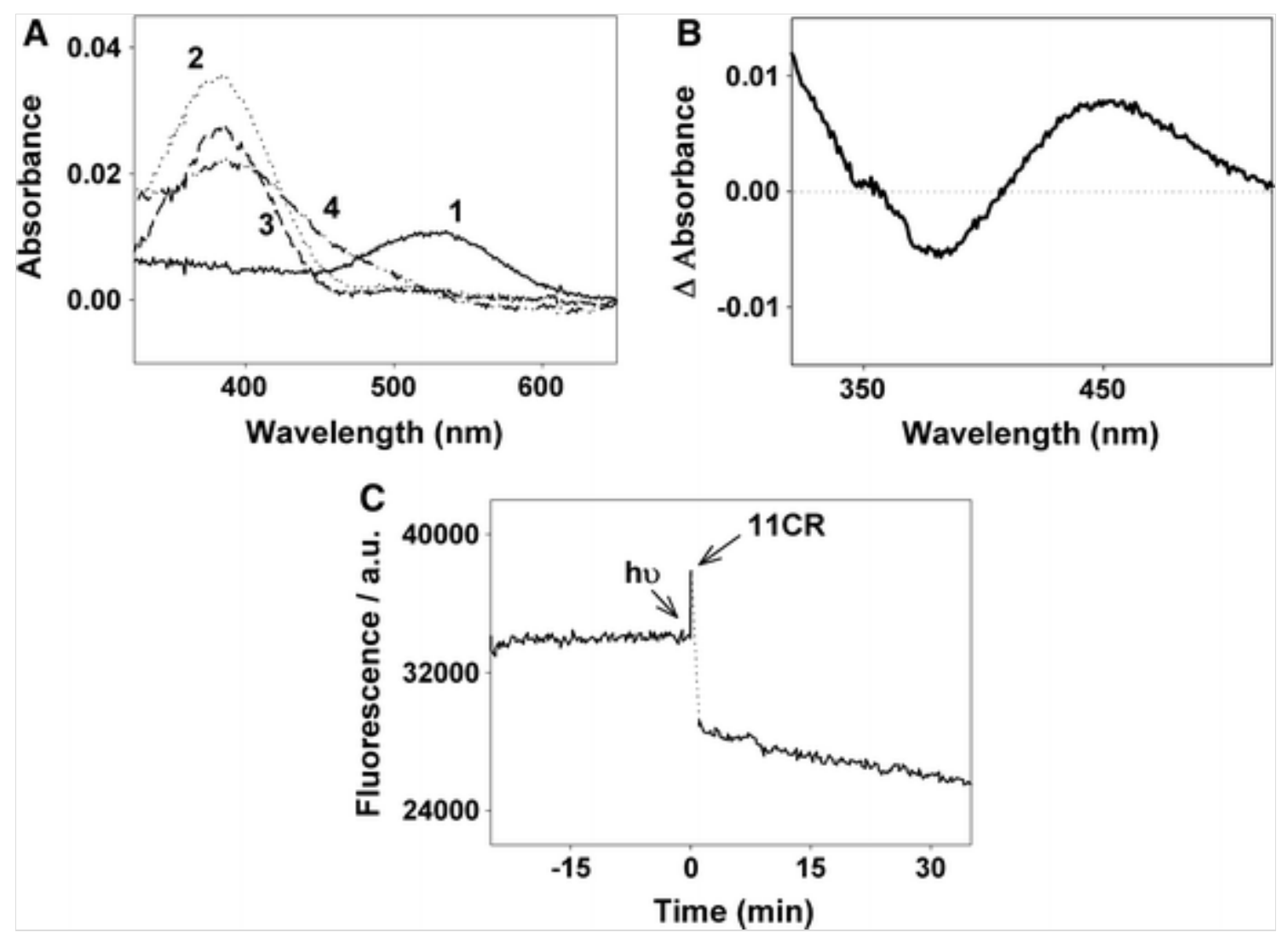

We also measured the photobleaching process by means of fluorescence spectroscopy by monitoring the Trp fluorescence increase upon green cone opsin illumination (see "MethodsMaterials and methods"). In the dark state, the fluorescence from Trp residues at the retinal binding pocket is quenched by the $\beta$-ionone group of the retinal. Upon photoactivation, the exit of retinal from opsin results in a fluorescence increase that (as the cone opsins exhibit a fast MetaII decay kinetics) reaches a maximum within a second [20, 30]. Purified green cone pigment sample was illuminated after obtaining a stable baseline and the sudden increase in fluorescence indicated fast retinal release and MetaII decay processes. Subsequently, immediate addition of exogenous $11 \mathrm{CR}$ to the illuminated sample resulted in a decrease in the fluorescence signal confirming binding of $11 \mathrm{CR}$ to photoactivated green cone opsin (Fig. 2c) as previously reported [20].

Molecular modeling was carried out to understand the structural features behind such remarkable functionally relevant differences between red and green cone opsins. Figure 3 a shows a sequence alignment between the human red and green cone opsins (including bovine rhodopsin as a reference), excluding the $\mathrm{N}$ - and $\mathrm{C}$-terminus (Fig. $3 \mathrm{a}$ ). The two photoreceptors have same exact number of residues and differ in only 15 residues, with most changes located in the central portion of the transmembrane (TM) helices 5 and 6 . The similarity is remarkable in the retinal binding pocket, where only position 
$309^{1.40}$ in TM7 is different (Tyr and Phe in red and green opsins, respectively). Assuming a similar movement of this residue with receptor activation, as observed in the crystal structures of rhodopsin, Tyr309 would form, in red cone opsin, a hydrogen bond with Thr201 in ECL2 (Fig. 3 b). This interaction, which is not possible in green cone opsin, could underlie the observed differences in spectral and retinal regeneration properties. The involvement of the 309 residue in spectral tuning of human red and green cone pigments has been previously reported [31].

\section{Fig. 3}

Phe309 regulates Schiff base protonation during 11CR regeneration of photoactivated green cone opsin. a Sequence alignment between the human red and green opsins and bovine rhodopsin (in gray), excluding the $\mathrm{N}$ - and $\mathrm{C}$-terminus. Residues highlighted in blue indicate differences between red and green opsins (numbered according to the general scheme [7]). b Molecular model of the inactive red opsin showing the proposed interaction between Tyr309 $9^{7.40}$ (in TM7) and Thr201 (in ECL2). The cartoon of TM7 and the conformation of Tyr309 in the active state are shown with a transparent representation, and the arrow indicates the movement. c $11 \mathrm{CR}$ regeneration experiment was carried out with purified F309Y green cone opsin mutant. After measuring a dark-state spectra (1), 11CR was added prior to illumination (2) and the regenerated spectrum was recorded after $30 \mathrm{~min}$ (3) showing a band at $530 \mathrm{~nm}$ and the sample was acidified (4). c inset. A closer look at the visible region of dark (1) and (2) regenerated spectra. d The difference spectrum, obtained by subtracting the acidified spectrum and the regenerated spectrum, shows a clear difference band at $530 \mathrm{~nm}$ 

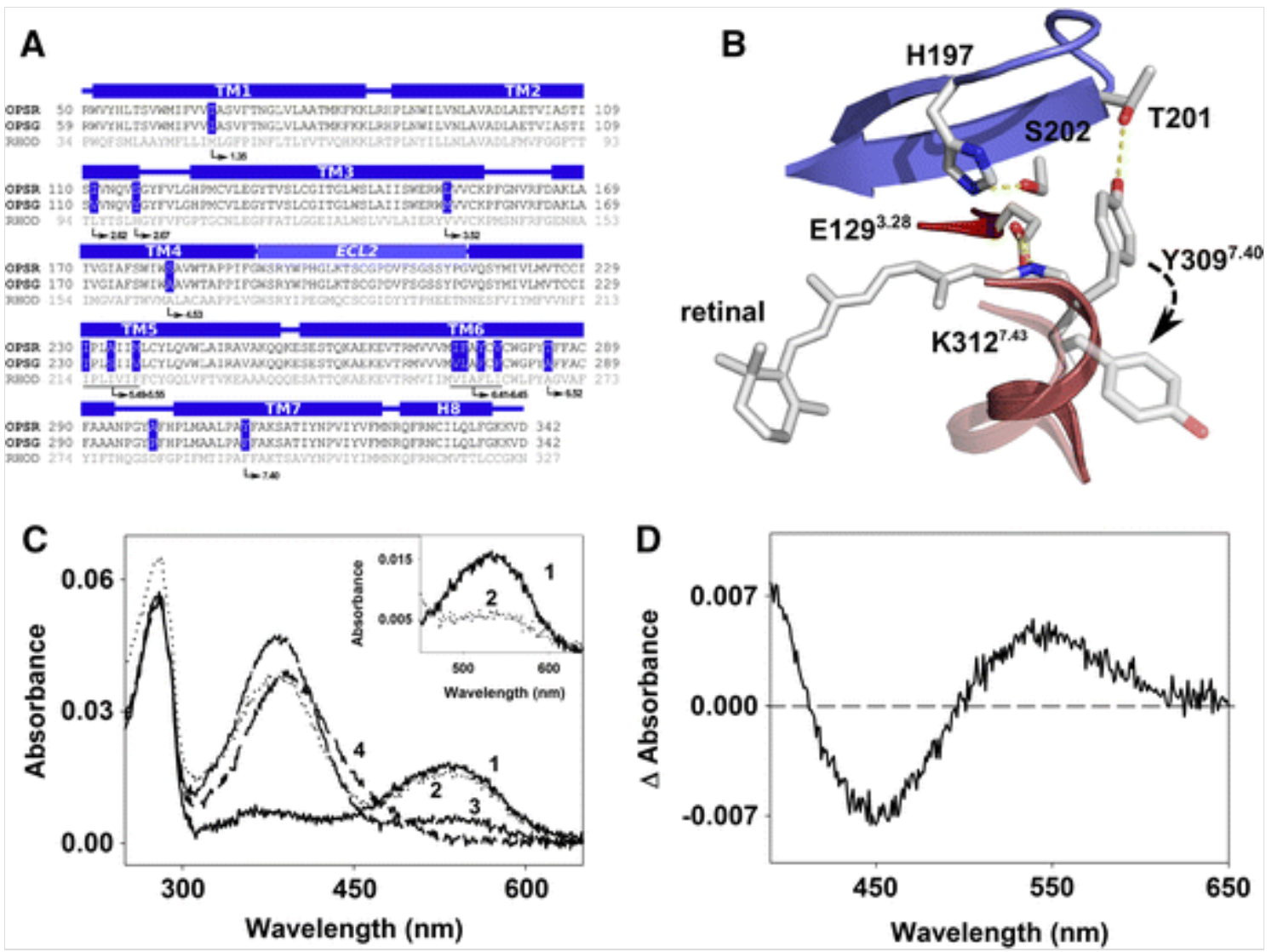

To validate the modeling observation, Phe309Tyr mutation was introduced into green cone opsin and the $11 \mathrm{CR}$ regeneration experiment was carried out with the purified mutant pigment which showed a regenerated peak at $530 \mathrm{~nm}$ (Fig. $3 \mathrm{c}$ ). The difference between the acidified and the regenerated spectra showed a clear band at $530 \mathrm{~nm}$ representing the PSB in 309Tyr mutant of green cone opsin, and the difference band at $440 \mathrm{~nm}$ corresponds to the amount of net SB from the regenerated pigment (Fig. $3 \mathrm{~d}$ ). The fact that the amplitude of the $530 \mathrm{~nm}$ band is lower than that of WT pigment regenerated with $11 \mathrm{CR}$, suggests that other residues, like Ala285 ${ }^{6.52}$ (Thr in red cone opsin), close to the $\beta$-ionone ring and Phe $277^{6.44}$ (Tyr in red cone opsin), close to $\operatorname{Trp} 288^{6.48}$ are responsible for the spectral differences in green and red opsins. However, the role of Tyr309 $9^{7.40}$ in modulating the process of protonation of photoactivated green cone pigment during 11CR regeneration has been substantiated.

\section{Red and green cone opsins chromophore regeneration kinetics}

The conformation of opsin and the photochemistry of the chromophore determine the spectral properties of cone visual pigments and, in turn, the observed spectral behavior could provide hints about the structural details of the receptor proteins [32]. Illumination causes a decrease in absorbance of the 
regenerated pigment band in the visible region which would be apparently parallel to an absorbance decrease at $380 \mathrm{~nm}$. The $380-\mathrm{nm}$ absorbance decrease, upon chromophore regeneration, could reflect either 11CR binding to, or ATR release from, the opsin receptor. Each data point corresponding to the absorption maximum in the visible region and the retinal region of each regenerated spectra was plotted versus time. The data could be fit to the curves reflecting a fast regeneration process that rapidly reached a plateau.

\section{Regeneration kinetics of green and red cone opsin with 9CR}

In red and green cone opsins, the kinetics of the 9CR regeneration process gave $t_{1 / 2}$ of $1.31 \pm 0.39 \mathrm{~min}$ and $0.66 \pm 0.23$ by red and green cone opsin, respectively (Fig. 4a). The decrease in absorbance at $380 \mathrm{~nm}$ parallels the visible increase and represents entry of retinal into photoactivated opsin during the regeneration process (Fig. 4b) as expected. Parallel experiments were carried out with purified rhodopsin, in which the regeneration with 11CR and 9CR followed single saturation patterns which are significantly slower than those observed for cone opsins, and can be correlated with absorbance changes at $380 \mathrm{~nm}$ (Fig. S3).

\section{Fig. 4}

Regeneration and retinal release kinetics of green and red cone opsin with 9CR. a The absorbance increase at the $\lambda_{\text {max }}$ in the visible region of each regenerated red/green pigment was plotted and fit with a saturation curve. Chromophore regeneration with 9CR was measured for red and green cone opsins for $30 \mathrm{~min}$. The dark-state spectral values of red and green cone opsin were normalized to have equivalent dark absorbance and the regenerated absorbances were also normalized by the same factor. b Absorbance changes at $380 \mathrm{~nm}$ for the regenerated samples were plotted and fit to a single exponential decay curve
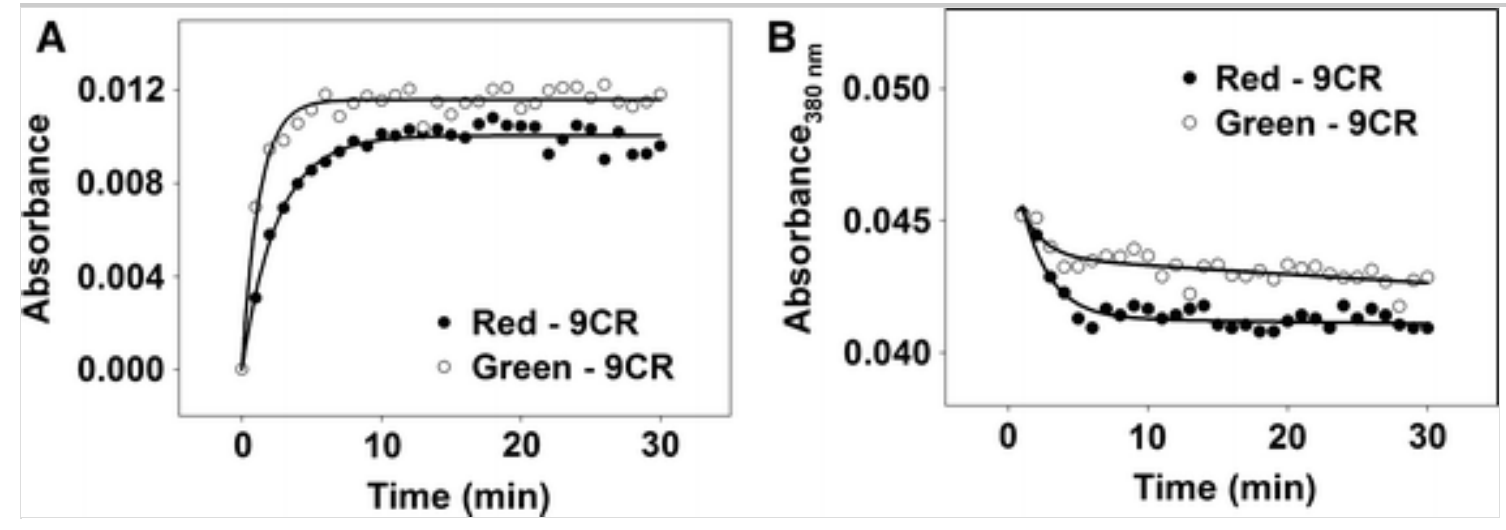

Secondary retinal uptake kinetics by cone visual pigments after regeneration 
Analogous regeneration experiments were carried out with 11CR but only for red cone opsin as green cone opsin showed no absorbance increase in the visible region upon regeneration. A similar pattern to that observed for $9 \mathrm{CR}$ was obtained in the case of red cone opsin regenerating with $11 \mathrm{CR}$ with a $t_{1 / 2}$ of $0.70 \pm 0.3 \mathrm{~min}$, and the regeneration with $11 \mathrm{CR}$ was found to be even faster than with 9CR (Fig. $5 \mathrm{a}$ ). This behavior is in agreement with the complete fast regeneration observed for red cone opsin with 11CR (Fig. 1 c).

\section{Fig. 5}

Regeneration and secondary retinal uptake kinetics of red and green cone opsin with $11 \mathrm{CR}$. a 2.5 -fold of $11 \mathrm{CR}$ to the concentration of red cone opsin was added in the dark and illuminated for $30 \mathrm{~s}(\lambda>495 \mathrm{~nm})$. UV-visible spectra of the sample were obtained every min for $30 \mathrm{~min}$. The $\lambda_{\max }$ at the visible region of each regenerated spectrum was plotted and fit with a saturation curve. $\mathbf{b}$ In the regeneration experiments with $11 \mathrm{CR}$, the regeneration was followed at $380 \mathrm{~nm}$ and the absorbance change at this wavelength was monitored for $30 \mathrm{~min}$ and the data fit to a sigmoidal curve. c Molecular model of the active red opsin showing the potential coexistence of two retinal molecules one in the primary site (as observed in the rhodopsin structure with PDB id 2X72 [49] in pale yellow) and another in the proposed secondary sites (showing representative poses obtained from flexible docking; in multiple colors). TM helices are represented as cylinders and side-chains of neighboring residues are shown with sticks 

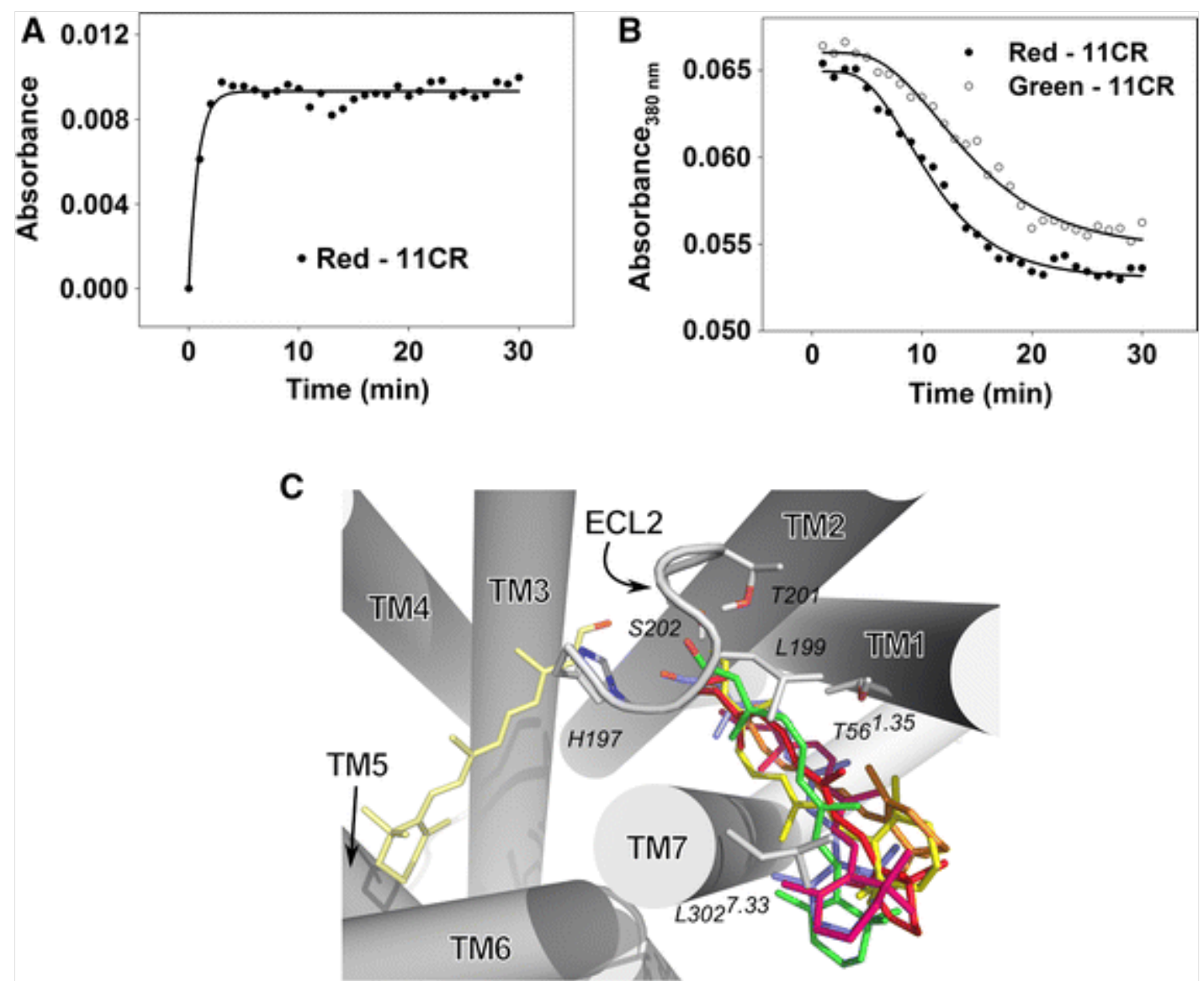

Cone opsin regeneration is usually quantified by measuring the absorbance increase at the characteristic visible band, but in our case this was not possible for green cone opsin chromophore regeneration with 11CR. In order to estimate the regeneration kinetics, we measured the decrease in absorbance at $380 \mathrm{~nm}$ with time. This would reflect retinal entry into the binding site of cone opsins and was expected to parallel the regeneration process. In this regard, the change in absorbance at $380 \mathrm{~nm}$ after illumination was plotted and the data could be unexpectedly fit to a sigmoidal curve (Fig. $5 \mathrm{~b}$ ) which suggested entry of $11 \mathrm{CR}$ into visual pigments at a slower rate, with $t_{1 / 2}$ of $11.38 \pm 2.34 \mathrm{~min}$ for red, and $13.12 \pm 0.78 \mathrm{~min}$ for green cone opsin, respectively. This behavior is in contrast to the faster binding kinetics of cone opsins regenerated with 11CR (Fig. $5 \mathrm{a}$ ). This fast regeneration which is complete within 1.0 min suggests that the slower process observed (Fig. $5 \mathrm{~b}$ ) cannot be due to retinal binding to the $11 \mathrm{CR}$ canonical pocket, but suggested additional uptake of $11 \mathrm{CR}$ into a secondary binding site facilitated by the presence of $11 \mathrm{CR}$ excess in the protein milieu.

In the search for a possible secondary binding pocket for $11 \mathrm{CR}$, we computed interaction potential maps for a hydrophobic probe in both inactive and active models of red and green opsins (see "Materials and methods"). The maps 
revealed a cavity between TMs 1 and 7 in the active models of red and green opsins as well as in rhodopsin with sufficient size to accommodate a second retinal molecule. Flexible docking calculations revealed possible binding modes of retinal penetrating through TMs 1 and 7 (Fig. $5 \mathrm{c}$ ) placing the aldehyde group of retinal close to His197, Thr201, Ser202 in ECL2 and the $\beta$-ionone ring in the mouth of the hydrophobic pocket between TM1 and TM7, flanked by residues Thr56 ${ }^{1.35}$ and Leu302 ${ }^{7.33}$. Because TM7 moves with activation, increasing the size of the cavity, only the active structures seem to allow binding of a second retinal in this region. This cavity is compatible with the proposed entry/exit channels for retinal in rhodopsin [33] and with the presence of hydrophobic molecules observed in the crystal structures of rhodopsin, as we previously noted [34].

AQ2

We interpret this phenomenon as a slower binding of a second 11CR molecule to the regenerated pigment to a site with lower affinity than that at the canonical ligand binding pocket (Fig. 6). This secondary 11CR uptake might be potentially coupled with a delay in ATR release from the protein. We propose that this slower secondary retinal uptake is a distinct phenomenon to that of the fast regeneration of red and green cone opsins observed in a $\sim 1$ min time frame with $11 \mathrm{CR}$. Such a behavior was not observed when photoactivated red and green cone opsins were regenerated with 9CR (Fig. 4b) suggesting that it is specific in the case of the native $11 \mathrm{CR}$ chromophore.

\section{Fig. 6}

Photoactivated intermediate conformations and $11 \mathrm{CR}$ regeneration of red and green cone pigments. a Cone pigments heterologously expressed in mammalian cells were regenerated with $11 \mathrm{CR}$ and immunopurified. b Exogenous 11CR (2.5 molar ratio over opsin) was added in the dark, to the immunopurified regenerated sample, and the sample was subsequently illuminated $(\lambda>495 \mathrm{~nm})$. This causes the isomerization of opsin-bound $11 \mathrm{CR}$ to ATR resulting in a protein conformational change - that This facilitates fast pre-binding of free $11 \mathrm{CR}$ to a secondary, lower affinity, retinal binding site followed by chromophore regeneration. c Fast chromophore regeneration would be achieved by translocation and covalent binding of this pre-bound $11 \mathrm{CR}$ to the chromophoric binding pocket by displacing ATR which in turn would move out from the protein. Further, the secondary uptake of $11 \mathrm{CR}$ by the visual pigment, with a slower kinetics, may be due to the conformational change of the regenerated protein coupled with the reduced availability of bulk 11CR that would synergistically decelerate $11 \mathrm{CR}$ binding to the secondary site. d Under 
our experimental conditions that would lead, at this stage, to a $11 \mathrm{CR}$-regenerated visual pigment harboring an additional $11 \mathrm{CR}$ bound to the postulated secondary binding site

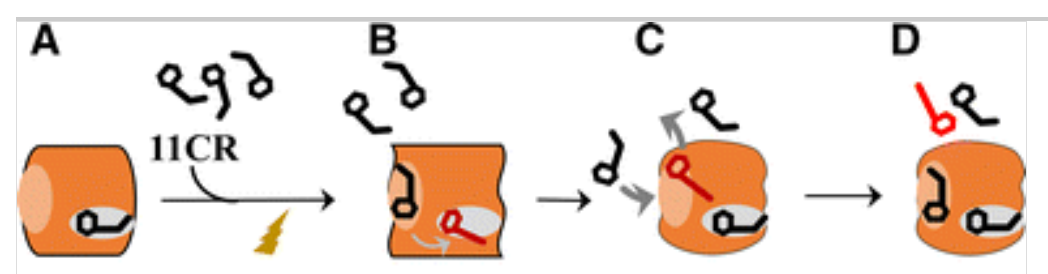

ho

\section{Discussion}

Specific amino acids at the retinal binding site, as well as their interactions with the retinal chromophore and surrounding water molecules, modulate spectral tuning of visual opsins [35-37]. Cone opsin pigments have been much less characterized than the rod photoreceptor rhodopsin due to their lower availability and their higher conformational instability. With only $4 \%$ variance between red and green cone opsin sequences, these were believed to have analogous structure-function relationships, other than the well-established wavelength absorption fine-tuning in the visible region of the spectrum.

Green cone opsin regenerates through an unprotonated SB intermediate conformation that eventually has to become protonated in vivo to regenerate the 530-nm absorbing chromophore. This novel intermediate conformation may have a decreased $\mathrm{pKa}$ of the SB nitrogen, in the case of green cone opsin, that could imply electrostatic changes at the SB linkage vicinity including changes in the SB-counterion distance. Though the mechanism that would convert this unprotonated SB intermediate conformation to the typical native green cone opsin pigment is unclear, the native membrane environment could potentiate the reaction by shortening the life-time of the transient intermediate we observe in our in vitro system. Green cone cells are less abundant in the human retina (the ratio of red cone cells to green cone cells in the human retina mosaic is estimated to be $1.5-11.5: 1)$ but their response is believed to be analogous to that of red cone cells [38]. This different cone cells population, and the structural differences herein reported, could be linked to specific subtle differential roles for red and green cone visual pigments in color vision.

A clear difference is found in the intermediate conformation after 
photoactivation of red and green cone opsin pigments that may be related to differences arisen in the molecular evolution of these two genetically closely related visual pigments. Analogous transient intermediate conformations of the ligand-free opsin, during the regeneration process, have also identified for rhodopsin [39]. The unprotonated SB conformation detected could be involved in the dark adaptation process of the photoactivated receptor, in an excess of retinal environment, and consequently play a role in the molecular basis of color vision by differentially modulating the chromophore regeneration and functionality of green and red cone photoreceptor cells.

We believe that our spectral changes are not reflecting potential retinal binding to other regions on the surface, or external side of the protein, for several reasons. First, the secondary binding observed is not covalent, so binding to external Lys residues is excluded. It should also be pointed out that retinal binding to the protein surface is unlikely to cause the spectral change at $380 \mathrm{~nm}$ observed. The absorbance decrease at $380 \mathrm{~nm}$ could be the result of an altered extinction coefficient resulting from the second retinal bound to hydrophobic helices and the extracellular domain of the receptor. This secondary retinal uptake, after retinal binding to its primary site, could be coupled with ATR exit from the regenerated cone opsins. The exit of the isomerized ATR could share the same channel as the entry of the secondary retinal molecular and this would justify the slower kinetics of the sigmoidal curves obtained (Fig. 5).

Furthermore, such non-specific retinal-protein surface interactions would not be isomer specific but, in our case, only $11 \mathrm{CR}$ can induce the spectral behavior observed. Thus, the secondary binding site proposed shows specificity towards $11 \mathrm{CR}$, but not 9CR, and such ligand specific accessibility would be difficult to explain if the secondary retinal binding would happen at the surface of the protein. The geometry of 11CR would favor accessibility into the opsin secondary binding site in contrast to the 9CR geometry. This would reflect that $11 \mathrm{CR}$ has been evolutionarily selected to act as the natural chromophore in vision. All these arguments reinforce our proposal of secondary retinal binding at the proposed site by our molecular modeling analysis. Notably, the two phase kinetics observed from the fluorescence regeneration experiment (Fig. 2c) in the case of green cone opsin provides further support to this interpretation. The fast component would represent regeneration with $11 \mathrm{CR}$ at the primary site, and the second slower component would be related to the secondary retinal uptake. 
Our findings are in agreement and support the results of previous studies proposing that more than one retinoid can interact with visual opsins [40] which strongly suggests the presence of functionally relevant secondary binding sites of retinoids in opsin [41]. Furthermore, previous experimental evidence showed an important role for non-covalent $11 \mathrm{CR}$ binding to opsin prior to regeneration [42] and in its ability to bind to a selective conformation of the protein among various conformational modes of visual pigments prior and after photoactivation [39].

In addition to the secondary $11 \mathrm{CR}$ binding event, changes at $380 \mathrm{~nm}$ could also be reflecting changes in ATR release that could alter the rate of retinoid recycling thus regulating dark-state adaptation after photoactivation for cone opsins. Retinoid binding protein, ABCA4, is an ATP dependent transporter of ATR from the luminal side to the cytoplasmic side of photoreceptor cells. At the cytoplasmic side, ATR is converted to all-trans-retinol by RDH8 which is then released to Müller cells or retinal pigment epithelium (for the retinoid cycle) and further converted to $11 \mathrm{CR}$ which in turn diffuses into photoreceptor cell and results in dark adaptation of visual pigments [43-45]. Stargardt disease is an autosomal recessive disorder characterized by mutations at ABCA4, in which vision loss is associated with accumulation of ATR and its derivatives that can be toxic by causing oxidative stress that can result in damage of the retinal cells [46, 47]. It is also known that transportation of ATR by ABCA4 is a multi-step process and that the substrate for ABCR4 is N-retinylidene-phosphotidyl ethanolamine (NRPE) formed from the interaction of ATR with phosphotidyl ethanolamine. NRPE is a reversible product, and the conversion process of RDH8 from ATR to all-trans-retinol is slow [43, 48]. Hence, the controlled release of ATR after photoactivation, coupled to $11 \mathrm{CR}$ binding to a secondary binding site other than the canonical retinal binding pocket, would help regulating the effective transport of ATR, from cone opsins. This may suggest a temporary entrapment of ATR to the same or other secondary binding site, and this may be connected to a slower movement of the hydrophobic retinal molecule along the ligand channel proposed between openings A and B which would be perpendicular to the helices axis. A secondary retinal binding site in cone visual pigments can accommodate a retinal molecule in addition to the one bound to the classical retinal binding pocket [40]. We propose that this second binding site (specific for $11 \mathrm{CR}$ and not for 9CR) may function as a retinal buffer, in cone opsins, and may possibly have an allosteric role although this may need further investigation. The different behavior for $11 \mathrm{CR}$ and $9 \mathrm{CR}$ is also evident 
from the faster initial velocity for the chromophore regeneration observed with 11CR than 9CR (Figs. 4, 5). This secondary binding site may allow retinal pre-binding and subsequent fast transfer of this retinal molecule to the primary retinal binding pocket for covalent binding and dark-adapted pigment regeneration ready for another light stimulus.

\section{Acknowledgments}

This work was supported by a Grant from Fundación Ramón Areces, and in part by Grants from the Ministerio de Ciencia e Innovación (Spain) (SAF2011-30216-C02-01) and by Grups de Recerca Consolidats de la Generalitat de Catalunya (2009 SGR 1402) (to P.G.), and by a CIG Grant from the European Commission (to E.R.). S.S. is the recipient of a FI-AGAUR Fellowship.

\section{Electronic supplementary material}

Below is the link to the electronic supplementary material.

Supplementary material 1 (DOCX $61253 \mathrm{~kb}$ )

\section{References}

1. Hartridge H (1946) Colour receptors of the human fovea. Nature 158:97

2. Thomson LC, Wright WD (1947) The colour sensitivity of the retina within the central fovea of man. J Physiol 105:316-331

3. Pearring JN, Salinas RY, Baker SA, Arshavsky VY (2013) Protein sorting, targeting and trafficking in photoreceptor cells. Prog Retin Eye Res 36:24-51. doi:10.1016/j.preteyeres.2013.03.002

4. Deeb SS (2005) The molecular basis of variation in human color vision. Clin Genet 67:369-377. doi:10.1111/j.1399-0004.2004.00343.x

5. Nathans J, Piantanida TP, Eddy RL, Shows TB, Hogness DS (1986) Molecular genetics of inherited variation in human color vision. Science 232:203-210

6. Shichida Y, Matsuyama T (2009) Evolution of opsins and 
phototransduction. Philos Trans R Soc Lond B Biol Sci 364:2881-2895. doi:10.1098/rstb.2009.0051

7. Ballesteros JA, Weinstein H (1995) Integrated methods for the construction of three-dimensional models and computational probing of structure-function relations in $\mathrm{G}$ protein-coupled receptors. Methods in Neurosciences 25:366-428

8. Hofmann KP, Scheerer P, Hildebrand PW, Choe HW, Park JH, Heck M, Ernst OP (2009) A G protein-coupled receptor at work: the rhodopsin model. Trends Biochem Sci 34:540-552. doi:10.1016/j.tibs.2009.07.005

9. Kakitani H, Kakitani T, Rodman H, Honig B (1985) On the mechanism of wavelength regulation in visual pigments. Photochem Photobiol 41:471-479

10. Loppnow GR, Barry BA, Mathies RA (1989) Why are blue visual pigments blue? A resonance Raman microprobe study. Proc Natl Acad Sci USA 86:1515-1518

11. Merbs SL, Nathans J (1992) Absorption spectra of human cone pigments. Nature 356:433-435. doi:10.1038/356433a0

12. Mustafi D, Engel AH, Palczewski K (2009) Structure of cone photoreceptors. Prog Retin Eye Res 28:289-302.

doi:10.1016/j.preteyeres.2009.05.003

13. Borhan B, Souto ML, Imai H, Shichida Y, Nakanishi K (2000) Movement of retinal along the visual transduction path. Science 288:2209-2212

14. Peters K, Applebury ML, Rentzepis PM (1977) Primary photochemical event in vision: proton translocation. Proc Natl Acad Sci USA 74:3119-3123

15. Honig B, Greenberg AD, Dinur U, Ebrey TG (1976) Visual-pigment spectra: implications of the protonation of the retinal Schiff base. Biochemistry 15:4593-4599

16. Matthews RG, Hubbard R, Brown PK, Wald G (1963) Tautomeric 
17. Zaitseva E, Brown MF, Vogel R (2010) Sequential rearrangement of interhelical networks upon rhodopsin activation in membranes: the Meta II(a) conformational substrate. J Am Chem Soc 132:4815-4821. doi:10.1021/ja910317a

18. Knierim B, Hofmann KP, Ernst OP, Hubbell WL (2007) Sequence of late molecular events in the activation of rhodopsin. Proc Natl Acad Sci USA 104:20290-20295. doi:10.1073/pnas.0710393104

19. Kubli-Garfias C, Salazar-Salinas K, Perez-Angel EC, Seminario JM (2011) Light activation of the isomerization and deprotonation of the protonated Schiff base retinal. J Mol Model 17:2539-2547. doi:10.1007/s00894-010-0927-x

20. Srinivasan S, Ramon E, Cordomi A, Garriga P (2014) Binding specificity of retinal analogs to photoactivated visual pigments suggest mechanism for fine-tuning GPCR-ligand interactions. Chem Biol 21:369-378. doi:10.1016/j.chembiol.2014.01.006

21. Crouch RK, Kono M (2014) Ligand control of g protein-coupled receptor activity: new insights. Chem Biol 21:309-310. doi:10.1016/j.chembiol.2014.03.001

22. Li J, Edwards PC, Burghammer M, Villa C, Schertler GF (2004) Structure of bovine rhodopsin in a trigonal crystal form. J Mol Biol 343:1409-1438. doi:10.1016/j.jmb.2004.08.090

23. Scheerer P, Park JH, Hildebrand PW, Kim YJ, Krauss N, Choe HW, Hofmann KP, Ernst OP (2008) Crystal structure of opsin in its G-proteininteracting conformation. Nature 455:497-502. doi:10.1038/nature07330

24. Sali A, Blundell TL (1993) Comparative protein modelling by satisfaction of spatial restraints. J Mol Biol 234:779-815.

doi:10.1006/jmbi.1993.1626

25. Case DAD TA, Cheatham TE III, Simmerling CL, Wang J, Duke RE, Luo R, Walker RC, Zhang W, Merz KM, Roberts B, Wang B, Hayik S, Roitberg A, Seabra G, Kolossváry I, Wong KF, Paesani F, Vanicek J, Liu J, 
Wu X, Brozell SR, Steinbrecher T, Gohlke H, Cai Q, Ye X, Wang J, Hsieh M-J, Cui G, Roe DR, Mathews DH, Seetin MG, Sagui C, Babin V, Luchko T, Gusarov S, Kovalenko A, Kollman PA (2011) AMBER 11, (University of California, San Francisco)

AQ3

26. Lindorff-Larsen K, Piana S, Palmo K, Maragakis P, Klepeis JL, Dror RO, Shaw DE (2010) Improved side-chain torsion potentials for the Amber ff99SB protein force field. Proteins 78:1950-1958.

doi:10.1002/prot. 22711

27. Goodford PJ (1985) A computational procedure for determining energetically favorable binding sites on biologically important macromolecules. J Med Chem 28:849-857

28. Trott O, Olson AJ (2010) AutoDock Vina: improving the speed and accuracy of docking with a new scoring function, efficient optimization, and multithreading. J Comput Chem 31:455-461. doi:10.1002/jcc.21334

29. Sakmar TP, Franke RR, Khorana HG (1991) The role of the retinylidene Schiff base counterion in rhodopsin in determining wavelength absorbance and Schiff base pKa. Proc Natl Acad Sci USA 88:3079-3083

30. Farrens DL, Khorana HG (1995) Structure and function in rhodopsin. Measurement of the rate of metarhodopsin II decay by fluorescence spectroscopy. J Biol Chem 270:5073-5076

31. Asenjo AB, Rim J, Oprian DD (1994) Molecular determinants of human $\mathrm{red} / \mathrm{green}$ color discrimination. Neuron 12:1131-1138

32. Blatz PE, Liebman PA (1973) Wavelength regulation in visual pigments. Exp Eye Res 17:573-580

33. Hildebrand PW, Scheerer P, Park JH, Choe HW, Piechnick R, Ernst OP, Hofmann KP, Heck M (2009) A ligand channel through the G protein coupled receptor opsin. PLoS ONE 4:e4382.

doi:10.1371/journal.pone.0004382

34. Ramon E, Cordomi A, Aguila M, Srinivasan S, Dong X, Moore AT, 
Webster AR, Cheetham ME, Garriga P (2014) Differential light-induced responses in sectorial inherited retinal degeneration. J Biol Chem 289:35918-35928. doi:10.1074/jbc.M114.609958

35. Sekharan S, Sugihara M, Buss V (2007) Origin of spectral tuning in rhodopsin - it is not the binding pocket. Angew Chem Int Ed Engl 46:269-271. doi:10.1002/anie.200603306

36. Palczewski K, Kumasaka T, Hori T, Behnke CA, Motoshima H, Fox BA, Le Trong I, Teller DC, Okada T, Stenkamp RE, Yamamoto M, Miyano M (2000) Crystal structure of rhodopsin: a G protein-coupled receptor. Science 289:739-745

37. Okada T, Fujiyoshi Y, Silow M, Navarro J, Landau EM, Shichida Y (2002) Functional role of internal water molecules in rhodopsin revealed by X-ray crystallography. Proc Natl Acad Sci USA 99:5982-5987. doi:10.1073/pnas.082666399

38. Sharpe LT, Stockman A, Jägle H, Nathans J (2001) Opsin genes, cone photopigments, color vision and color blindness. In: Gegenfurther KR, Sharpe LT (eds) Color vision: from genes to perception. Cambridge University Press, Cambridge

39. Schafer CT, Farrens DL (2015) Conformational selection and equilibrium governs the ability of retinals to bind opsin. J Biol Chem 290:4304-4318. doi:10.1074/jbc.M114.603134

40. Makino CL, Riley CK, Looney J, Crouch RK, Okada T (2010) Binding of more than one retinoid to visual opsins. Biophys $\mathrm{J}$ 99:2366-2373. doi:10.1016/j.bpj.2010.08.003

41. Heck M, Schadel SA, Maretzki D, Hofmann KP (2003) Secondary binding sites of retinoids in opsin: characterization and role in regeneration. Vision Res 43:3003-3010

42. Kefalov VJ, Crouch RK, Cornwall MC (2001) Role of noncovalent binding of 11-cis-retinal to opsin in dark adaptation of rod and cone photoreceptors. Neuron 29:749-755

43. Tsybovsky Y, Molday RS, Palczewski K (2010) The ATP-binding 
cassette transporter ABCA4: structural and functional properties and role in retinal disease. Adv Exp Med Biol 703:105-125.

doi:10.1007/978-1-4419-5635-4_8

44. Maeda A, Maeda T, Sun W, Zhang H, Baehr W, Palczewski K (2007) Redundant and unique roles of retinol dehydrogenases in the mouse retina. Proc Natl Acad Sci USA 104:19565-19570.

doi:10.1073/pnas.0707477104

45. Lamb TD, Pugh EN Jr (2004) Dark adaptation and the retinoid cycle of vision. Prog Retin Eye Res 23:307-380.

doi:10.1016/j.preteyeres.2004.03.001

46. Allikmets R, Singh N, Sun H, Shroyer NF, Hutchinson A, Chidambaram A, Gerrard B, Baird L, Stauffer D, Peiffer A, Rattner A, Smallwood P, Li Y, Anderson KL, Lewis RA, Nathans J, Leppert M, Dean M, Lupski JR (1997) A photoreceptor cell-specific ATP-binding transporter gene $(\mathrm{ABCR})$ is mutated in recessive Stargardt macular dystrophy. Nat Genet 15:236-246. doi:10.1038/ng0397-236

47. Chen Y, Ratnam K, Sundquist SM, Lujan B, Ayyagari R, Gudiseva VH, Roorda A, Duncan JL (2011) Cone photoreceptor abnormalities correlate with vision loss in patients with Stargardt disease. Invest Ophthalmol Vis Sci 52:3281-3292. doi:10.1167/iovs.10-6538

48. Molday RS (2007) ATP-binding cassette transporter ABCA4: molecular properties and role in vision and macular degeneration. $\mathrm{J}$ Bioenerg Biomembr 39:507-517. doi:10.1007/s10863-007-9118-6

49. Standfuss J, Edwards PC, D’Antona A, Fransen M, Xie G, Oprian DD, Schertler GF (2011) The structural basis of agonist-induced activation in constitutively active rhodopsin. Nature 471:656-660.

doi:10.1038/nature09795 